\title{
TOWARDS A VIRTUAL ENTERPRISE FOR PASSENGER TRANSPORTATION USING AGENTS
}

\author{
Claudio Cubillos ${ }^{1}$, Franco Guidi-Polanco ${ }^{2}$ and Claudio Demartini ${ }^{3}$

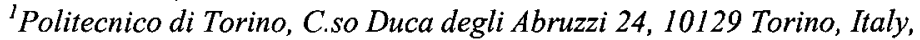 \\ e-mail: claudio.cubillos@polito.it \\ ${ }^{2}$ Pontificia Universidad Católica de Valparaíso, Av. Brazil 224I, Valparaíso, Chile, \\ e-mail:fguidi@ucv.cl \\ ${ }^{3}$ Politecnico di Torino, C.so Duca degli Abruzzi 24, 10129 Torino, Italy, \\ e-mail: claudio.demartini@polito.it
}

\begin{abstract}
This work presents a framework for developing a virtual enterprise for passenger transportation using the agent paradigm as baseline. The framework provides a set of base agents and behaviours that give the basic service functionality. The agent architecture allows the integration of different fleet operators and vehicle providers on one side, and users with their different groups and requirements on the other. A Service Ontology uniforms the semantics for building customized transport descriptions and for formalizing the interaction among the different actors and agents. An implementation focused in the centralized planification of the trips requests has been done using Jade and their results are presented.
\end{abstract}

\section{INTRODUCTION}

Nowadays flexible passenger transportation systems, also known as demand responsive transport (DRT), promise a greater flexibility for the user, a wider range of destinations and an increased opportunity for mode shift as potential benefits.

However, these benefits can get lost due to lack of integration. The spatial and administrative distribution of the different transport providers operating on specific areas (whether metropolitan or rural) often determines a fragmented coverage of the user needs. This results in poor customer service provision and in negative feedbacks for the authorities. Therefore, DRT transportation solutions need to be efficiently coordinated between them and with the other transport solutions. As a result, the trend is to move from a group of individual DRT systems towards a single virtual transport provision enterprise, a Virtual Mobility Agency.

On the other hand, the agent technology has been successfully applied to the transport domain and to system-integration scenarios (Zhang et al, 2003). Therefore, this work extends our research (Cubillos et al, 2004) on a single operator transportation framework to a virtual enterprise scenario that integrates different vehicle providers, using the agent paradigm as baseline. 


\section{TRANSPORTATION REQUIREMENTS}

In a complex service system like passenger transportation, there are many actors that have a direct interest in the commercial, social and infrastructural impacts of the services. Four main Actor Categories can be identified, each of which with several subgroups. The categories are:

End Users: Are the direct or potential customers of the provided transport service. They can also be described as the "passenger" or "consumer". Commonly DRT services are considered most important among the following End User groups: the elderly, the disabled, healthcare patients (trips to health centers), school children and students, shoppers/visitors and persons with no access to a car.

Transport Operators: The Operator is directly involved in the provision of the transport service to the End User by providing some or all of the service elements such as the vehicle, driver, dispatch and support services.

Active Destinations: Certain destinations may play an active role in arranging transportation for their customers. For example, they may supply information to the operator about trips to the destination or they may order them for their customers; they may assist the operator in planning the services, or they may co-operate with the operator to provide a reasonable price for the trips. For example, hospitals, shopping centers and several civil service departments are typically this kind.

These actors usually have different and sometimes conflicting needs. Users commonly specify transport requests with different characteristics (pick-up/delivery place/time, low floor, WC, Air Conditioning, etc). On the other hand, single vehicle providers and fleet operators offer different transportation alternatives such as busses, minivans, vehicles for disabled people and taxis among others, each of them with diverse characteristics. Therefore, the flexible transport system we are treating consists of requests coming from a set of clients which should be satisfied by such an heterogeneous fleet.

From a mathematical point of view the problem corresponds to the dynamic version of the Dial-a-ride Problem (D-DARP) in a multi-depot scenario. This dynamicity means that the vehicle progress is monitored; clients can modify or cancel their trip requests, vehicle delays can occur, clients may not show up at the pickup place and vehicles can breakdown among others, involving the re-scheduling of the trips and their management.

\section{THE VIRTUAL TRANSPORTATION ENTERPRISE}

The framework provides a set of base agents and behaviours that provide the basic service functionality. As Figure 1 shows, an agent architecture allows the integration of the different transportation operators and vehicle providers on one side, and users with their different groups and requirements on the other. In addition, an Enterprise Service Ontology uniforms the semantics for building customized transport descriptions and for formalizing the interaction among the different actors and agents (service provision). The architecture relies over the Jade agent platform (Bellifemine, 1999) that provides a distributed environment with the basic communication and management services for the agents to interact.

First are explained the single vehicle and user parties, while in a next section is explained the integration of operators and user associations. 


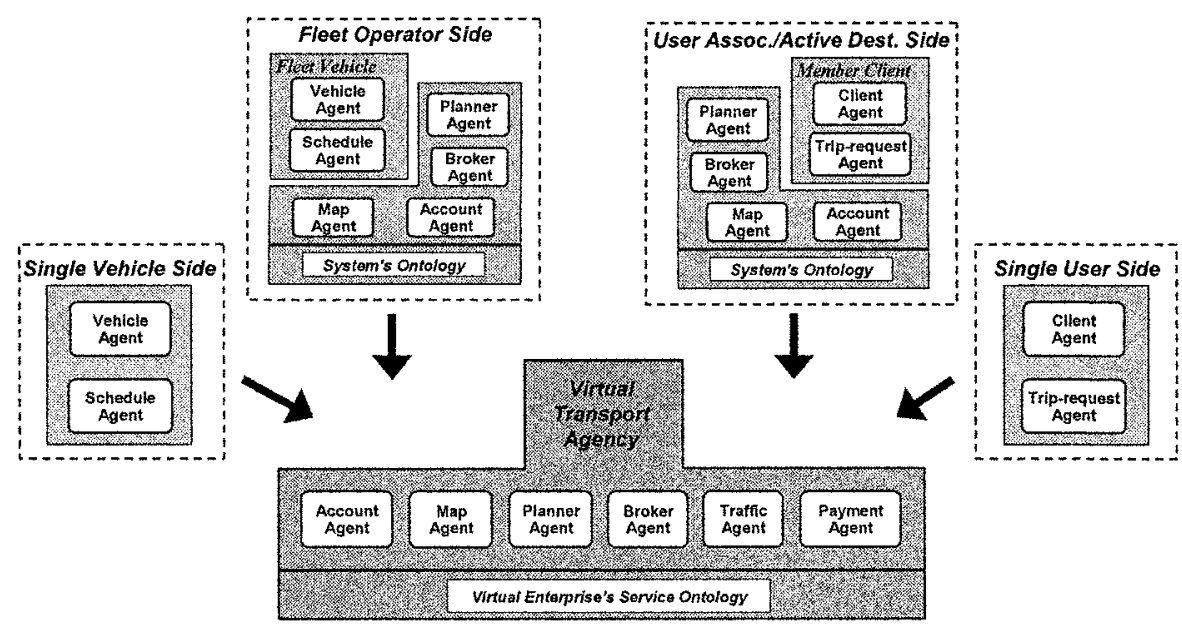

Figure 1 - Virtual Transportation Enterprise model.

\subsection{Single User Side}

Individual clients and their requirements are captured by Client and Trip-request agents. Together they provide full communication and interoperability of the real end user with the transportation system. The Client agent is in charge of providing a personalized user interface while the Trip-request manages the process of requesting the service, its characteristics and the decision making required.

The interface provided by Client agents should be adaptable to the different devices that can be used by clients for establishing the communication. Client agents can reside on different devices such as cell phones, PDAs or PCs, allowing offering the end user a pervasive interaction with the transport service. In addition, the Client agent is responsible for capturing all the client's requirements not only concerning the desired type of transport service but also his preferences upon contingency situations (e.g. delays, traffic jams, deviations, etc). The Trip-request takes these requirements and preferences to act on behalf of the real client during the whole process. Depending on the degree of autonomy provided by the client, the Triprequest can act as a personal trip assistant or simply as a mere proxy of the client decisions.

\subsection{Single Vehicle Side}

Each real vehicle is represented by an agent couple, the Vehicle and the Schedule agents. They provide interoperability between the vehicle they represent and the transportation system to which they belong to. The Vehicle agent plays an interface role, providing the vehicle and its driver with a communication channel with the rest of the transportation system. Through it, the driver is able to communicate along the journey about any contingency that could arise. From the other side, the driver receives through the Vehicle agent any information regarding changes on the original planification such as new client to transport, client cancellations and relevant traffic conditions changes among others. The Schedule agent is in charge of 
managing the vehicle's route and processing any new request for client transportation.

The Vehicle agent is also responsible for monitoring and informing about the vehicle state and progress of the service along the day. In a complementary way, the Schedule agent is responsible for any adjustments needed on the vehicle's route or schedule of their trips, motivated by any change or eventuality.

Both agent couples, Vehicle - Schedule and Client - Trip-request, can be developed independently by each involved party. The agent architecture defines the base roles for these agents to implement together with providing a common platform for enabling the communication. The Service Ontology defines without ambiguity the terms and concepts required for the agent understanding. In addition, this common ontology provides the formalization of the interaction protocols required by agents to communicate. In this way, any of the above agents can be constructed externally by implementing the functionalities required for its roles following the semantics and interaction specifications provided.

\subsection{Transportation Enterprise Side}

The transportation service role is mainly carried out by the Planner agent acting as a front face to the clients. Besides it there is a whole set of service agents collaborating to give support to the different required functions, such as the matching of request to vehicles (Broker Agent), the geographical data access (Map Agent), the accountability of the transactions (Account Agent) and the service payment (Payment Agent) among others.

From them, the most critical ones from the planning and control point of view are the Broker and the Map agents. The Broker is the one in charge of carrying out the matching of transport requests with available vehicles. For that is able to manage service descriptions coming from both sides, understand their semantics and perform the search.

The Map agent represents the geographic area giving information about localization of addresses and stops, street names and distances between localizations among others. For this purpose, the Map can perfectly act as a wrapper of a Geographical Information System (GIS). An important feature is the possibility of complement the information provided by the GIS with current traffic data. This can be done by a Traffic agent that is connected to different traffic information services and is able to inform about relevant events occurring in the streets and highways being covered. In this way, the system can consider those eventualities and re-plan the initial scheduling of vehicles to best adapt to the new situation.

Other complementary services can be "agentified" within the system. A service that can be put under this category is the collection and management of the information related to the fares, timetables, schedules and service characteristics of each of the involved vehicles. In this way, this information can be given to the interested users and organizations.

These supporting services can be externalized, that is, other third parties can interact with the enterprise in order to provide those services from the outside. In this sense an important thing to highlight is that as the enterprise's Service Ontology defines the valid interactions among agents and hence formalizes the way of providing any support service. Then, any party wishing to supply a service within 
the framework has to implement the respective agent adopting the specification given by the ontology. For example, a party that wants to offer the service of vehicle-request matching within the organization has to implement a Broker agent able of processing the specific query messages and of registering the available vehicles as defined by the ontology. Refer to (Cubillos et al, 2004) for further details on the ontology.

In this way, B2B relations are established among transport operators and support-service providers. For example, the geographical and traffic information services are used internally by the planning agents and the accountability services are required by the Planner to register the transactions that are carried out. In contrast, some of the services imply a direct request and benefit of the client. In these cases, B2C relations are created. For example, the information on traffic, timetables and fares provided to the clients goes under the $\mathrm{B} 2 \mathrm{C}$ service category.

\section{FLEET OPERATORS AND USER GROUPS INTEGRATION}

Up to now have been identified the three main actors. In addition to them there are other parties associated to the problem that would be important to consider. These are the user associations, the active destinations and fleet providers or operators.

Each fleet operators needs an own planning and control system for working. As Figure 1 shows at the up-left, each transport operator's system can be modeled within our architecture in a recursive way. In other words, each operator can have his system conformed by agents for their vehicles monitoring, integrated planning and supporting services. All this sustained by a local ontology that provides common service concepts and interaction formalizations. In this way, the operator's agents are capable of interoperation with the virtual enterprise by using their local ontology for the internal communication, while using the common enterprise ontology for the external communication with the virtual enterprise or a third party.

An analogous situation happens with the integration of the different client associations and active destinations. As Figure 1 shows on the up-right, they can also be modeled following a similar model as the one used for the operators. Clients pertaining to an association or active destination are able to access the virtual transportation system by passing through their local systems. In this way, the preferential terms agreed with the association can be used by their members. This mechanism enforces the adaptation of the service offer to the different client demands. Again in this case, the association's system uses a local ontology used by their agents for the internal coordination and communication while the common enterprise ontology is used for the external interactions.

\subsection{Wrapper Agents}

Assuming the similar agent architecture for the different parties (operators, user associations and active destinations) enforces a straightforward integration of them within the virtual enterprise. However, this assumption is rather strict as many of them will have their own (and perhaps legacy) systems already working. In these cases, their integration through wrapper agents is the right move to follow. 
Each party's system can be easily integrated into the common architecture by using agents for wrapping their functionalities and enabling the interoperation with our virtual enterprise model. In this way, each wrap agent plays the role of translating some functionality from the party's local system (with a particular way of working, processing and interact) to the common enterprise framework defined by its service ontology. From that point on, the way of interaction between the wrapper agents and the enterprise ones is similar to the previous case with local agent systems.

\subsection{Hierarchical Interaction}

The interaction of the Planner and Service agents across the different sides (Fleet Operators, User Associations, Transport enterprise) is ruled by the formalized interactions (Service Ontology) and follows a hierarchical/federated model.

In particular, the interaction between the fleet operator (or user associations / active destinations) agents and the virtual enterprise ones follows a one-to-one relation between agents of each side, that is, planner with planner, broker with broker and so on, in such a way to conform a hierarchical/federated structure. For example let's analyze the Planner agents' interaction. The enterprise Planner will receive a transportation request from a client and in order to answer it needs to check the possibilities available. Therefore, it will query all the Planners of the registered fleet operators, collecting the alternatives and prepare a proposal.

\section{BASE IMPLEMENTATION}

Up to now have been described a transportation framework and its underlying agent architecture. In this section is described the basic planning functionalities and the default implementation provided to the different agents involved in the model considering the single vehicle and user sides. Similar extensions can be made to the fleet operator, user associations and active destination sides.

The underlying planning problem is treated in a distributed way following a twophased model. The former is an optimization phase, aimed to the identification of trip solutions that maximize the operator's utility, subject to the (client) request restrictions. This involves the Planner and Schedule agents. The latter is a negotiation phase that pursues an agreement between the transportation proposals and the client's interests. This is done by the Planner and the Trip-request agent.

\subsection{Generic Planning Sequence}

The dynamics of the generic planning sequence is shown at Figure 2: In step 1 (Schedule-me), for each transportation request from a Client a Trip-request agent asks the Planner to process it. The Planner processes the request (step 2) first by obtaining from the Broker the vehicles that match the required profile, and then (step 3) by making a call for trip-proposals to all the corresponding Schedule agents.

They send back their proposals and then the Planner (step 4) selects the most suitable alternatives among the received trip proposals by applying filtering policies and starts a negotiation process with the client (through its Trip-request agent). After 
arriving to agreement the Planner (step 5) tells the Schedule agent that won the proposal to add the trip to its actual schedule and tells the others their proposal rejection.

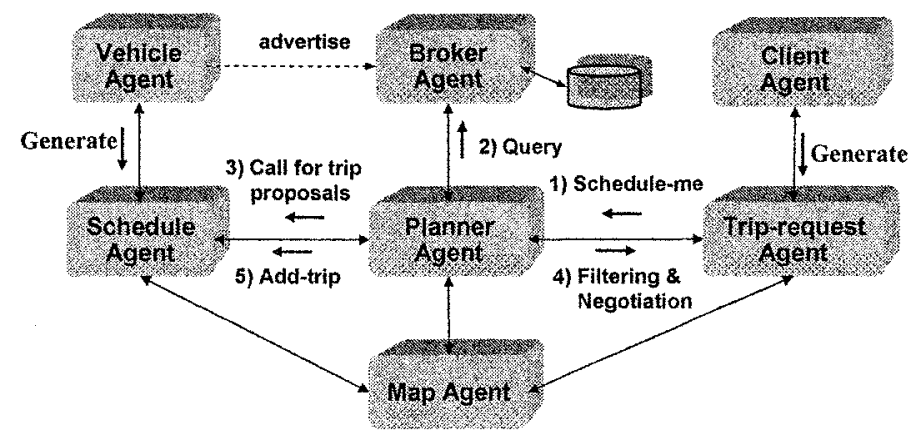

Figure 2 - The planning sequence

In the real world, clients can miss, cancel or modify the ride at last minute. The agents are able to deal with these situations and re-plan. As already mentioned, the route progress of each real vehicle is monitored by its Vehicle and Schedule agent. Upon differences in the planification (due to breakdowns, traffic jam, etc) the Schedule agent re-plans. In the case of having an infeasible trip request (mainly due to the time-window restrictions), it informs the Planner agent about the situation.

The Planner makes a call for trip-proposals to try reallocating the request in other available vehicle. In any case, the result is informed to the corresponding Triprequest agent, which depending on its degree of autonomy will process the alternatives and take a decision or will inform the client about the change. This change may imply a different vehicle processing the trip only or also a delay or an anticipation of the pickup and delivery times defined previously.

\subsection{Tests}

In order to check the correctness of the framework, a concrete system was built to provide a centralized scheme of planification. This centralized model was confronted with ADARTW (Jaw et al, 1986), a traditional centralized greedy insertion heuristic. Both systems minimize the number of used vehicles and were set with the same parameters.

The following operational decisions were adopted for both models: 1) the same utility function and scheduling algorithm were used for all the vehicles, 2) all the clients share the same utility function, 3) the available fleet is of 30 identical vehicles with capacity 20,4 ) one depot is used for all the vehicles and 5) in all cases the effectiveness measures (utility variables) were weighted with value 1 .

All the tests considered the same geographical net and 20 demand scenarios with 50 trip requests each, distributed uniformly in a two-hour horizon. For each demand scenario 25 runs were done. The Table 1 shows that the mean values for the different effectiveness measures are similar on both cases. Therefore, our centralized planification model extended from the transport framework performs in a similar way to ADARTW system. 
Table 1 - Centralized v/s ADARTW

\begin{tabular}{lccccccc} 
& $\begin{array}{l}\text { Serv. } \\
\text { Users }\end{array}$ & $\begin{array}{c}\text { Used. } \\
\text { Veh }\end{array}$ & Cost & $\begin{array}{c}\text { Wait } \\
\text { Time } \\
\text { [min] }\end{array}$ & $\begin{array}{c}\text { Excess } \\
\text { Time } \\
\text { [min] }\end{array}$ & $\begin{array}{c}\text { Bus } \\
\text { Travel } \\
\text { Time } \\
{[m i n]}\end{array}$ & $\begin{array}{c}\text { Slack } \\
\text { Time } \\
\text { [min] }\end{array}$ \\
\hline \multicolumn{2}{l}{ Centralized } & & & & & & \\
Mean & 50 & 27 & 1.687 & 23 & 9 & 1.655 & 72 \\
Desv.Est & 0 & 2 & 115 & 6 & 5 & 117 & 27 \\
Min & 47 & 21 & 1.412 & 6 & 0 & 1.383 & 20 \\
Max & 50 & 30 & 2.008 & 41 & 22 & 1.984 & 157 \\
\hline ADARTW & & & & & & & \\
Mean & 50 & 27 & 1.688 & 23 & 9 & 1.585 & 72 \\
Desv.Est & 0 & 2 & 113 & 6 & 6 & 119 & 28 \\
Min & 48 & 20 & 1.394 & 4 & 0 & 1.280 & 19 \\
Max & 50 & 30 & 2.001 & 41 & 28 & 1.912 & 170 \\
\hline & & & & & & & \\
\hline
\end{tabular}

The main difference between both systems is in the processing time. ADARTW takes 1 second in processing the run with 50 requests, while our agent-based model takes 20 seconds running on 1 machine. This allows to have an idea of the overhead introduced by two things: 1) the agent platform (Jade), and 2) the agent architecture.

A following step is to implement different planification models and provide a test bed for distributed execution.

\section{CONCLUSIONS}

The present work has described a transportation architecture that has been developed thinking in the independent implementation (externalization) of all the roles and functionalities involved, by providing a common framework that formalizes and enables the relations between the different parties. It is able to broaden along the whole transportation chain and is flexible enough to incorporate new actors.

In this way a true virtual transportation agency is provided, capable of putting together different groups and types of transport providers, end users and supportservice providers interested in using (or being part of) an integrated gateway for flexible people mobility.

\section{REFERENCES}

1. Bellifemine, F. et al.. "JADE - A FIPA Compliant Agent Framework". CSELT Internal Technical Report, 1999. Available at: http://jade.cselt.it/

2. Cubillos, C.; Guidi-Polanco, F.; Demartini, C. "Multi-Agent Infrastructure for Distributed Planning of Demand-Responsive Passenger Transportation Service". IEEE Int. Conf. on SMC, 2004, pp. 2013 $-2017$.

3. Jaw, J.; Odoni, A.R.; Psaraftis H.N.; Wilson, N.H.M. "A Heuristic Algorithm for the Multi-Vehicle Advance-Request Dial-a-Ride Problem with Time Windows". Transportation Research B, Vol. 20B, No. 3, 1986, pp. 243-257.

4. Zhang Jin; Wang Hui; Li Ping; "Towards the applications of multi-agent techniques in intelligent transportation systems". Proc. IEEE Int. Conf. on ITS, Oct. 2003, Vol. 2, pp. 1750-1754. 